\title{
A MULTILAYER GROUNDWATER SAMPLER FOR CHARACTERIZING CONTAMINANT PLUMES
}

\section{FINAL REPORT}

December 18, 1992

\author{
EDWARD KAPLAN \\ RADIOLOGICAL SCIENCES DIVISION \\ DEPARTMENT OF NUCLEAR ENERGY \\ BROOKHAVEN NATIONAL LABORATORY \\ UPTON, NEW YORK 11973-5000
}

The submitted manuscript has been authored under Contract No. DE-AC02-76CH00016 with the U.S. Department of Energy. 
A Multilayer Groundwater Sampler for Characterizing Contaminant Plumes

\author{
Final Report
}

\author{
Edward Kaplan and John Heiser \\ Radiological Sciences Division \\ Department of Nuclear Energy \\ Brookhaven National Laboratory \\ Upton, New York 11973-5000
}

\begin{abstract}
This final report describes activities related to the design and initial demonstration of a passive multilayer groundwater sampling system. The apparatus consists of remotely controlled cylinders filled with deionized water which are connected in tandem. Vertical fine structure of contaminants are easily defined. Using the apparatus in several wells may lead to three dimensional depictions of groundwater contamination, thereby providing the information necessary for site characterization and remediation.
\end{abstract}

\title{
1.0 Introduction
}

This report describes activities covering the design, construction, laboratory and field testing of a multilayer groundwater sampler. A first prototype was made of glass and operated on the principle of controlled diffusion through a membrane which was used to control the rate at which the sampler would close. Unfortunately, this device proved unreliable. A new prototype was constructed of stainless steel and Teflon ${ }^{1}$, and was capable of being opened and closed remotely from the surface. Through laboratory tests and field trials, this second design has been found to reliably collect samples of groundwater simultaneously at more than one depth in a single well.

\subsection{Original Design}

A sampler was first designed based on the principle that diffusion through a membrane could be controlled in such a manner that the unit would close after a predetermined time. The device was made of glass and consisted of two parts. The top was a cylinder covered at one end with a membrane. The other end screwed into a plug. Inside, a well buoyancy would hold this part above the lower section. The bottom section was a bottle into which the top unit would drop as water diffused into the cylinder through the membrane. Their bottoms were removed and were fitted with Teflon rings to hold pure cellulose acetate and reconstituted cellulose (dialysis) membranes. Tiny holes were drilled in their sides near the threads. An internally threaded cylindrical glass connector ( $50 \mathrm{~mm}$ diameter) was modified into an approximate $500 \mathrm{ml}$ sample collection bottle. The top of a standard Teflon plug was machined out and threaded to accept the septum jar. Figures 1-2 show this original design.

\subsection{Laboratory Experiments}

Several laboratory experiments were performed in order to determine the time it would take for sufficient water to diffuse into the septum jar in such a manner that the top assembly would fall into

1 Teflon is a registered trademark of E.I. DuPont de Nemours \& Co. 
the sample collection bottle. Two holes of approximately $0.75 \mathrm{~mm}$ diameter were drilled into the septum jar, and a 0.45 micron $(\mu \mathrm{m})$ cellulose acetate membrane was used. The prototype was then lowered into a $2000 \mathrm{ml}$ graduated cylinder (Figure 3). In the first experiment, it took approximately 96 hours for the top assembly to completely move into the bottom section.

These experiments were continued using membranes of different pore sizes, and using other septum jars with different numbers and diameters of drilled holes in their sides. The objective was to find an optimum combination of membrane pore size and septum jar hole number/diameters, such that the top assembly would reliably close into the sample collection jar in about $48-72$ hours. In one experiment, it was found that a Spectrapor dialyzer tubing membrane (molecular weight cutoff approximately 12,000-14,000) allowed closure in about $72-96$ hours. These results were not reproducible. (See Table 1.)

Closure first occurred after about 18 hours in other experiments using $1.2 \mu \mathrm{m}$ membranes. A repetition of this experiment resulted in closure after three days. A specially designed $1.59 \mathrm{~mm}(1 / 16$ inch) diameter bit was used to drill two holes into a septum jar; closure occurred after 3 days. The experiment was repeated using a new $4.76 \mathrm{~mm}(3 / 16 \mathrm{inch})$ bit since closure was desired after no more than 3 days. The septum jar closed between 1-9 days in experiments using these holes. (See Table 1.)

\subsection{Results}

Experiments were continued using membranes of different pore sizes. Results could not be reproduced consistently for any combination of hole diameter or membrane pore size. In addition, the septum jars used in this prototype were poorly manufactured and often broke during modification. Attempts were made to correct this problem by using research-grade Pyrex jars. It was decided to redesign the sampler, after many unsuccessful trials, in order to overcome the difficulties encountered during laboratory experiments.

\subsection{New Design}

A prototype was constructed using Teflon and stainless steel (see Figures 4-7). This apparatus resembled a bailer or coliwassa, with several important differences: it operated remotely (manually, at present, and electronically, in the future); several units are placed in tandem to obtain simultaneous samples at different depths; units are filled with deionized water, lowered into place and opened, allowing equilibration with water in the wellbore, then closed and retrieved. Operation in this manner avoids degassing on the way up, and allows retricval of larger sample volumes of both dissolved and particulate matter. Stainless steel push-pull cables were used to open and close the units remotely.

End-caps of the existing prototype could be reduced in size for use in a standard 2-inch well, although use at Brookhaven National Laboratory (BNL) is in a 4-inch casing. Each sample size is 500 $\mathrm{mL}$. Future designs may use Teflon to produce lighter weight apparatus, although the performance of Teflon during decontamination will have to be determined.

\subsection{Laboratory Experiments}

Experiments were performed to determine the time for deionized water inside the unit to equilibrate with water outside the unit via diffusion. In the first set of experiments, a sampler was filled with a solution of methylene blue dye, then closed and placed inside a 2-liter graduated cylinder filled with deionized water. It opened after about 12 hours. The time it took for the dye inside to completely 
diffuse into the graduated cylinder was determined by taking periodic measurements of transmitted licki.. with a Bausch and Lomb Spectronic20 spectrophotometer.

Two such experiments were performed (see Table 2). Using a power law approximation, it was estimated that the sampler took about 24 hours for about $95 \%$ equilibrium to become established (see Figure 8). During this experiment, it was observed that a well-defined column of dye was noted coming out the bottom of the unit, with no dye coming from the top. This is indicative that the physical mixing mechanism was not strictly diffusive, at least in this confined space and stagnant environment.

A second set of experiments were performed to examine the performance of the sampler when surrounded by a larger volume of water. In these experiments, a sampler was filled with deionized water and lowered into a large container filled with 14 liters of tap water of known conductivity. A Yellow Springs Instrument Co. Model 32 conductance meter was connected to a Bausch \& Lomb Omniscribe strip chart recorder. It took about 18 minutes to obtain about $90 \%$ equilibration, with almost total equilibration in about 1 hour. (Sce Table 3 and Figure 9.) (The small depression starting at about 1.5 hours and lasting for about 1 hour is attributed to a temperature phenomenon because the building was closed during this period, and the laboratory temperature decreased overnight.)

\subsection{Field Trials}

Two field tests of the apparatus were conducted during July 1990 inside an existing BNL monitoring well. Previous studies have indicated that the phreatic aquifer is contaminated with various organic substances, primarily deriving from fuels (e.g., denser organics such as chloroform, trichloroethane, trichloroethylene, and tetrachloroethylene, as well as the light organics ethylbenzene, toluene, and various xylenes) [Day, et. al., 1986; Miltenberger, et. al., 1988]. The well is fully screened in the saturated zone and has a dedicated submersible pump. It was decided to lower a single sampler first, in order to gain experience avoiding any interference with tubing and pump inside the casing, and to determine whether the sampler would work properly. The sampler was easily lowered into the well without disturbing existing equipment. It was positioned such that the top of the upper sampler was about 0.3 meter (one ft.) below the water table (which was measured at 9.6 meters ( $31.6 \mathrm{ft}$.) using a Johnson Watermarker), and allowed to remain in place overnight, after which time it was removed, taken to the laboratory, examined and cleaned. Measurements of conductivity in both the aquifer and sampler (before and after insertion in the well) confirmed that the unit was watertight.

Another field trial was made during October 1990. Two tandem samplers filled with deionized water were lowered into the monitoring well just below the water table (measured at 9.9 meters (32.4 $\mathrm{ft}$.) using a Johnson Watermarker). The units were separated by about 0.91 meter ( $3.0 \mathrm{feet}$ ) and left in the well overnight. Prior to insertion into the well, a ground water sample was taken using standard BNL Safety and Environment Protection Division practices, that is, using the dedicated submersible pump in the well to purge the equivalent of ten bore volumes before taking a sample. This sample volume was transferred in the field into two $20-\mathrm{mL}$ vials for analysis of organics, and the remaining amount was filtered and collected for analysis of inorganic species.

Eighteen hours after they were placed into the well, the samplers were opened remotely from the surface, and left in this opened position for 75 minutes. Then, the units were closed and gently raised to the surface. Next, their contents were transferred as described previously. Immediately thereafter, the samplers were rinsed and refilled with deionized water and again, lowered into the well to the same depth as before. This time the units were kept open for about one minute during which they were raised and lowered 10 times for about 6 inches per stroke. The units were then closed, raised to the surface, and their contents transferred to collection bottles. This second experiment was 
performed to see if samples could be taken quickly by relying on forced displacement, rather than diffusion alone.

A second field trial meant to replicate results of the first field experiment was scheduled for the beginning of 1991. In December 1989 BNL was declared a SUPERFUND site. The well which was used for this project is part of BNL's sampling program for compliance with regulatory requirements under the SUPERFUND announcement. Intensive monitoring began just after our first sampling event. Dedicated submersible pumps were installed in wells in the sampling area, including the subject well. In addition, the remedial investigation and feasibility study process made it difficult to gain access to the well. After much difficulty, a second set of ficld samplings were scheduled for March-April 1992.

\subsection{Results}

Results of the first field sampling are shown in Figure 10. There are four measurements in each cluster. The bar at the right results from using a submersible pump (10 bore volumes removed prior to sampling). Two sets of samples were taken. In the first, the samplers were kept opened in the well for about one hour. The two middle bars resulted from this. Afterwards, the samplers were reinserted into the well and samples were obtained after about one minute, during which time the unit was moved about 6 inches up-and-down for 10 strokes. Upper and lower measurements for this sampler were averaged, with results shown in the bar at the far left.

Figure 10 indicates several interesting observations. First, our results are compatible with what was found in 1988 using a membrane sampler developed at the Wcizmann Institute of Science in Israel [Kaplan, et. al., 1991]. Next, all measurements taken with the new sampler were significantly higher than those taken via standard BNL pumped-sample procedures. Lastly, it appears that the units require about one hour or so in an opened position in order to obtain a representative sample of water at the depths of interest.

Figure 11 illustrates results obtained during the second field sampling episode. While a significant amount of time elapsed since the first field samples were taken, no remedial activities had taken place at the site. Comparing Figures 10 and 11, it is clear that the multilayer apparatus performed comparably, obtaining samples which were again significantly higher than those taken via standard sampling protocols.

\subsection{Discussion}

The large volume, remotely operated, mechanical sampling system as developed for this project, has been shown to provide groundwater samples simultaneously from two depths in the same well, without disturbing the aquifer being measured. In static laboratory tests, the system was shown to reach equilibrium with its environment in approximately one hour. The system was used in the field to sample near the water table of an aquifer contaminated with both light and dense organics derived from various fuels. In two separate field tests, it was shown that the system returned samples which were significantly greater than those obtained using submersible pumps operated conventionally, and that concentrations from separate depths were discernibly different from one another.

When used in several wells at the same site, the mechanical sampling system should be uniquely able to provide a three dimensional perspective on the spatial distribution of all types of contaminants dissolved constituents, non-aqueous phase liquids (light and dense), as well as suspended and colloidal particles. It is well known that microbes exist in aquifers which biodegrade various 
organic contaminants, and that these microbes preferentially exist attached to particulate matter. Therefore, it is clear that the mechanical sampler has an important role in the study of subsurface microbial remediation, unlike various membrane devices which return sedimeni-free samples.

The proof-of-concept phase has been successfully completed. An important activity which must now commence is the process by which the multilevel mechanical sampler can obtain acceptance for various environmental permitting activities. The Environmental Protection Agency is in the unique position to commission a rigorous study which could lead to the acceptance of the device and to its eventual commercialization.

\subsection{Miscellaneous}

A manuscript describing our experiences with the Weizmann membrane sampler was written by the team of individuals involved with its use at BNL in 1988. This group included our project officer at the time (E. Koglin). It was submitted to the editor of the Joumnal of Ground Water, and was accepted for publication in the March-A pril 1991 issue. [Kaplan, et. al., 1991]

A commercially available device was discovered which closely resembles the design for a one of our samplers. Patented by Arts Manufacturing and Supply Co. (AMS), Inc. (American Falls, Idaho), the device is sold for use in sampling tanks and wells only at one depth. Technical conversations were held with the owners of AMS, who agreed to furnish a sampler for laboratory testing. This was accomplished after project funds had been depleted. It was determined that the device's operating characteristics were quite similar to our design, and discussions began for using several of the AMS samplers remotely, in tandem, in a manner consistent with our design: that is, filled with deionized water and closed, lowered to required depths, allowed to sit, then opened for a period of time, closed, and returned to the surface. Funds were obtained to demonstrate the new configuration from the Department of Energy, Office of Technology Development. This activity is now in progress.

\subsection{References:}

Day, L.E., R.P. Miltenberger, and J.R. Naidu, eds., Environmental Monitoring Report, Brookhaven National Laboratory, BNL Report 51993 (1986).

Kaplan, E., et al, Multilayer Sampling in the Water-Table Region of a Sandy Aquifer, Ground Water, V. 29, Number 2, pp. 191-198 (1991).

Miltenberger, R.P., B.A. Royce, and J.R. Naidu, eds, Environmental Monitoring Report, Brookhaven National Laboratory, BNL Report 52152 (1988). 
Table 1 -- Experiments with original (i.e., glass) sampler to determine optimal design (i.e., number and size of holes; type and thickness of membranes).

\begin{tabular}{|c|c|c|c|c|}
\hline $\begin{array}{l}\text { Number of } \\
\text { Holes }\end{array}$ & $\begin{array}{l}\text { Hole Size } \\
(\mathrm{mm})\end{array}$ & $\begin{array}{l}\text { Membrane } \\
\text { Type }\end{array}$ & $\begin{array}{l}\text { Membrane } \\
\text { Thickness } \\
\text { (microns) }\end{array}$ & $\begin{array}{l}\text { Time to } \\
\text { Closure } \\
\text { (days) }\end{array}$ \\
\hline 0 & --. & Cellulose Acetate & 0.45 & $4^{1}$ \\
\hline 0 & $-\cdots$ & Cellulose Acetate & 0.45 & $7^{2}$ \\
\hline 2 & 0.75 & Regenerated Cellulose & 0.45 & 3 \\
\hline 4 & 0.75 & Regenerated Cellulose $^{3}$ &.- .4 & 3.5 \\
\hline 4 & 0.75 & Regenerated Cellulose 3 & $\ldots-.4$ & 4 \\
\hline 4 & 0.75 & Cellulose Acetate & 1.2 & 0.7 \\
\hline 4 & 0.75 & Cellulose Acetate & 1.2 & 3 \\
\hline 2 & 1.6 & Cellulose Acetate & 1.2 & 3 \\
\hline 2 & 1.6 & Cellulose Acetate & 1.2 & 9 \\
\hline 2 & 4.8 & Cellulose Acetate & 1.2 & 5 \\
\hline 2 & 4.8 & Cellulose Acetate & 1.2 & 4 \\
\hline 2 & 4.8 & Cellulose Acetate & 1.2 & 2 \\
\hline 2 & 4.8 & Cellulose Acetate & 1.2 & 2 \\
\hline 2 & 1.6 & Cellulose Acetate & 1.2 & 0.8 \\
\hline 2 & 1.6 & Cellulose Acetate & 1.2 & 1 \\
\hline 2 & 1.6 & Cellulose Acctate & 1.2 & 1.25 \\
\hline
\end{tabular}

1 No closure at end of experiment

2 No closure at end of experiment

3 Regenerated cellulose membrane from split dialyzer tubing

${ }^{4}$ Approximate $12 \mathrm{~K}-14 \mathrm{~K}$ molecular wcight cutoff 
Table 2 -. Time for second sampler design to equilibrate (using methyl blue dye as indicator).

\begin{tabular}{|c|c|c|c|}
\hline $\begin{array}{c}\text { Elapsed } \\
\text { Time } \\
\text { (min) }\end{array}$ & $\begin{array}{c}\text { Elapsed } \\
\text { Time } \\
\text { (hr) }\end{array}$ & $\begin{array}{c}\text { Measured } \\
\text { Trans } \\
\text { Run 1 }\end{array}$ & $\begin{array}{c}\text { Measured } \\
\text { Trans } \\
\text { Run } 2\end{array}$ \\
\hline ............ & - & -........ & -..--.-- \\
\hline 0 & 0.00 & 98.0 & 88.0 \\
\hline 30 & 0.50 & 91.5 & \\
\hline 60 & 1.00 & 87.0 & \\
\hline 75 & 1.25 & & 80.5 \\
\hline 90 & 1.50 & 86.5 & \\
\hline 120 & 2.00 & 85.0 & \\
\hline 150 & 2.50 & 84.0 & \\
\hline 180 & 3.00 & 82.0 & \\
\hline 315 & 5.25 & & 76.5 \\
\hline 420 & 7.00 & & 75.5 \\
\hline 1245 & 20.75 & 77.5 & \\
\hline 1440 & $24: 00$ & 77.5 & \\
\hline 1660 & 27.75 & 77.0 & \\
\hline 5760 & 96.00 & 78.0 & \\
\hline
\end{tabular}

page 8 
Table 3 .- Time for second sampler design to equilibrate (using conductance as indicator).

\begin{tabular}{|c|c|c|c|}
\hline $\begin{array}{c}\text { Elapsed } \\
\text { Time } \\
\text { (min) }\end{array}$ & $\begin{array}{c}\text { Elapsed } \\
\text { Time } \\
\text { (hr) }\end{array}$ & $\begin{array}{c}\text { Measured } \\
\text { Trans } \\
\text { Run } 1\end{array}$ & $\begin{array}{c}\text { Mcasured } \\
\text { Trans } \\
\text { Run } 2\end{array}$ \\
\hline -............ & -.......... & - & - \\
\hline 0 & 0.00 & 0.25 & 0.25 \\
\hline 10 & 0.17 & 6.0 & 5.6 \\
\hline 15 & 0.25 & 7.6 & 6.6 \\
\hline 20 & 0.33 & 8.6 & 8.1 \\
\hline 30 & 0.50 & 9.2 & 9.1 \\
\hline 40 & 0.67 & 9.5 & 9.2 \\
\hline 50 & 0.83 & 9.6 & 9.2 \\
\hline 60 & 1.00 & 9.7 & \\
\hline 90 & 1.50 & 9.6 & \\
\hline 120 & 2.00 & 9.4 & \\
\hline 180 & 3.00 & 9.8 & - \\
\hline
\end{tabular}



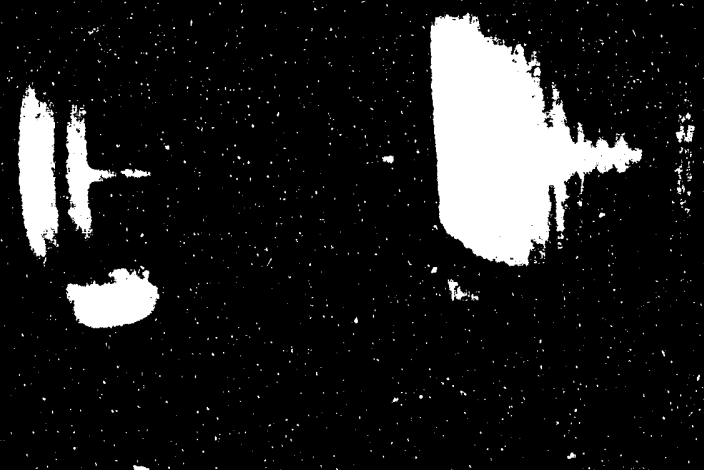


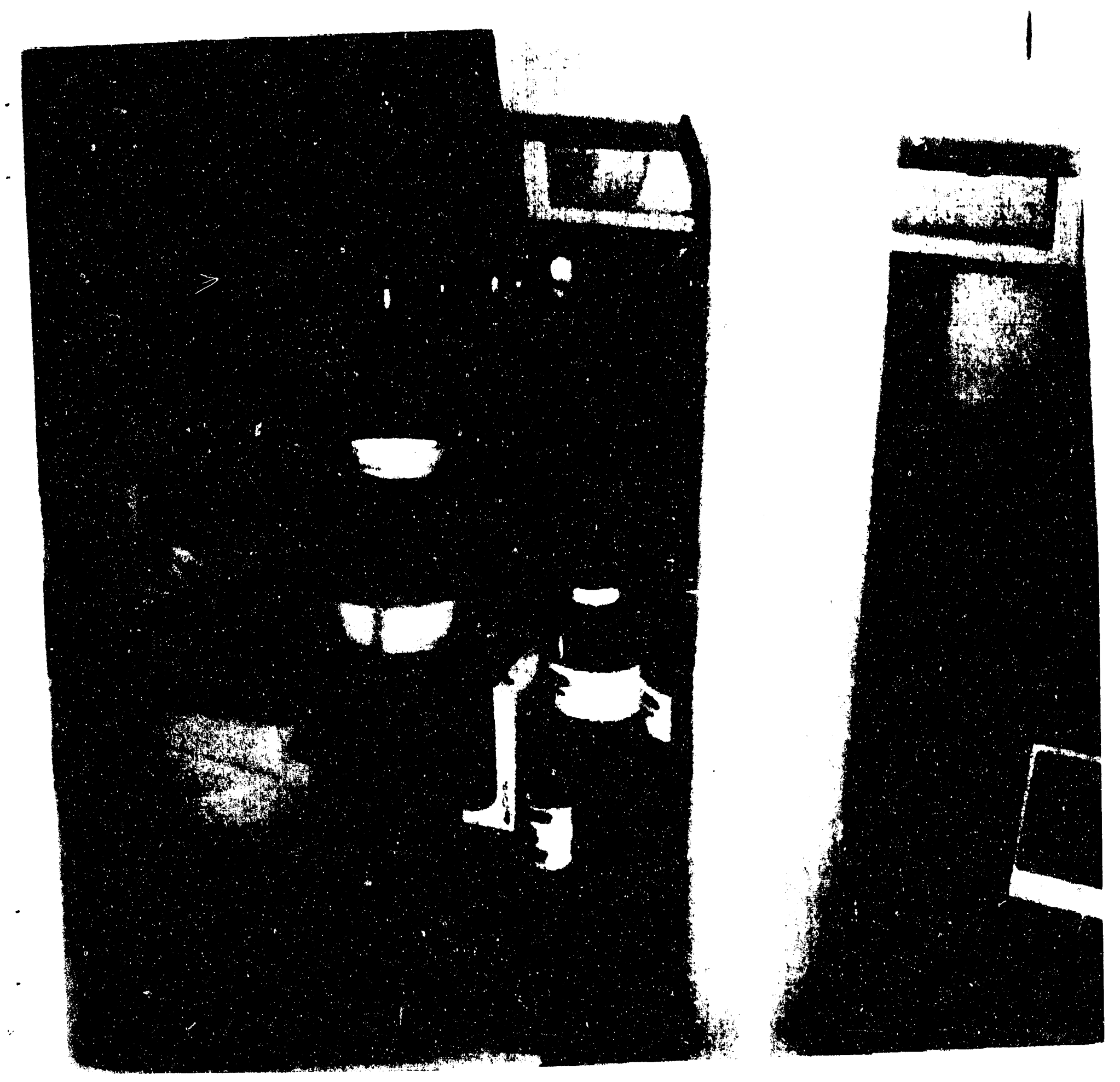

Figure 3 - Original Design of Sampler During Tests for Time to Equilibration. 


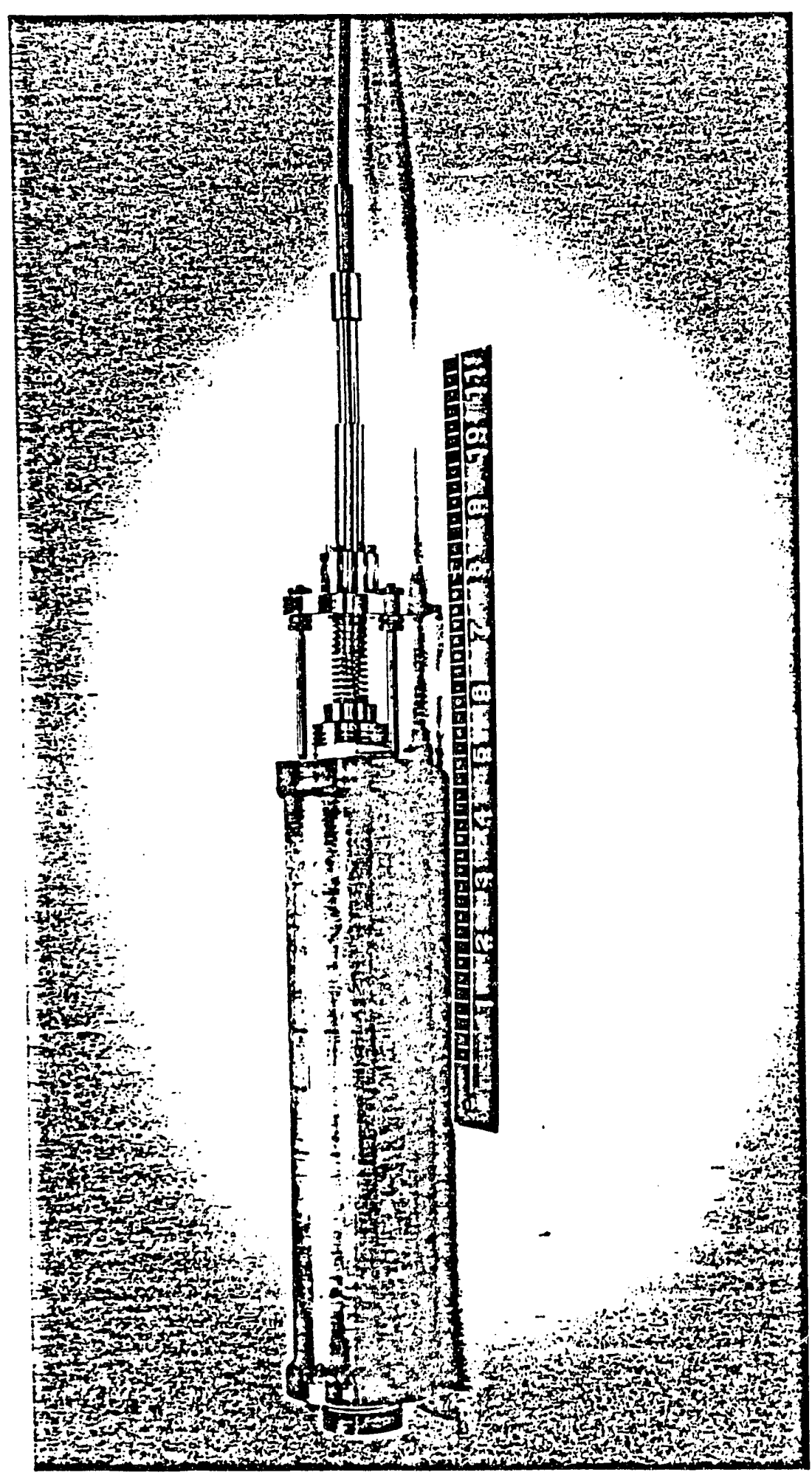

Figure 4 - Prototype mechanical sampler (single unit). 


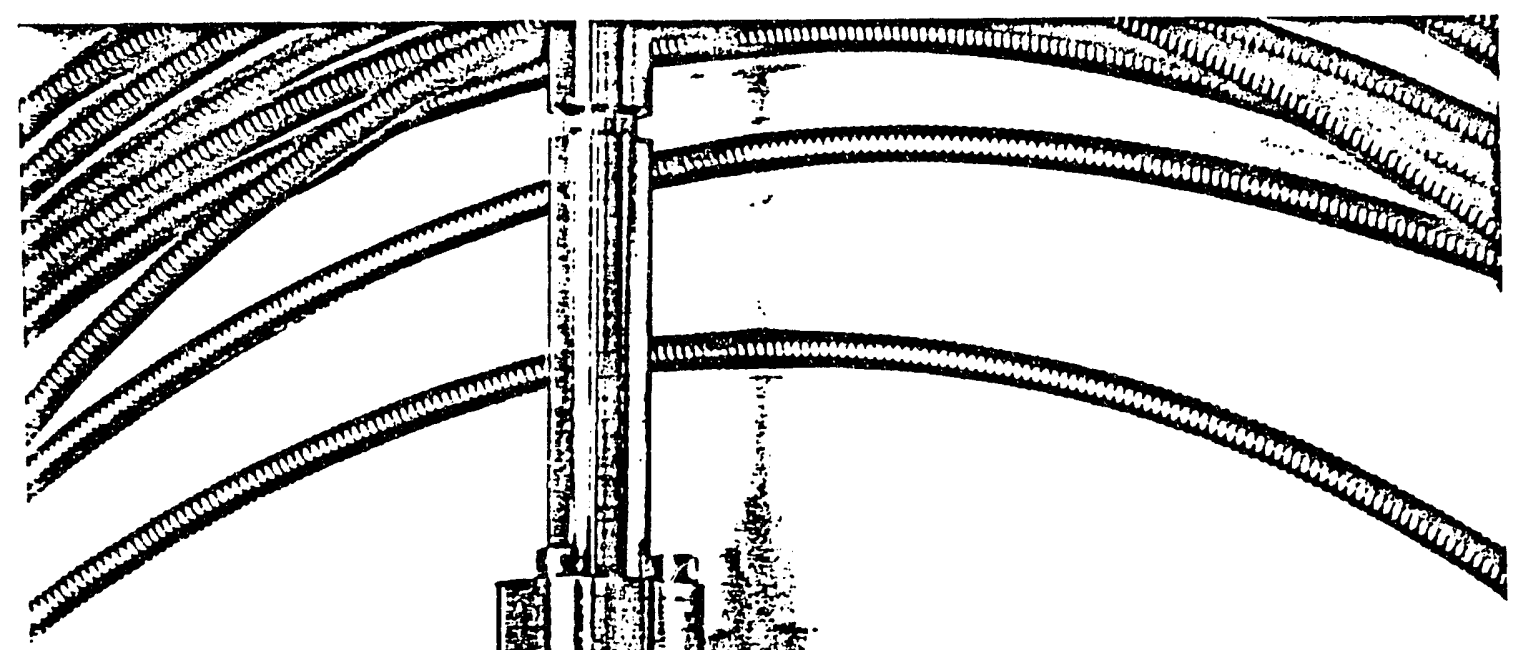

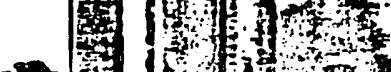

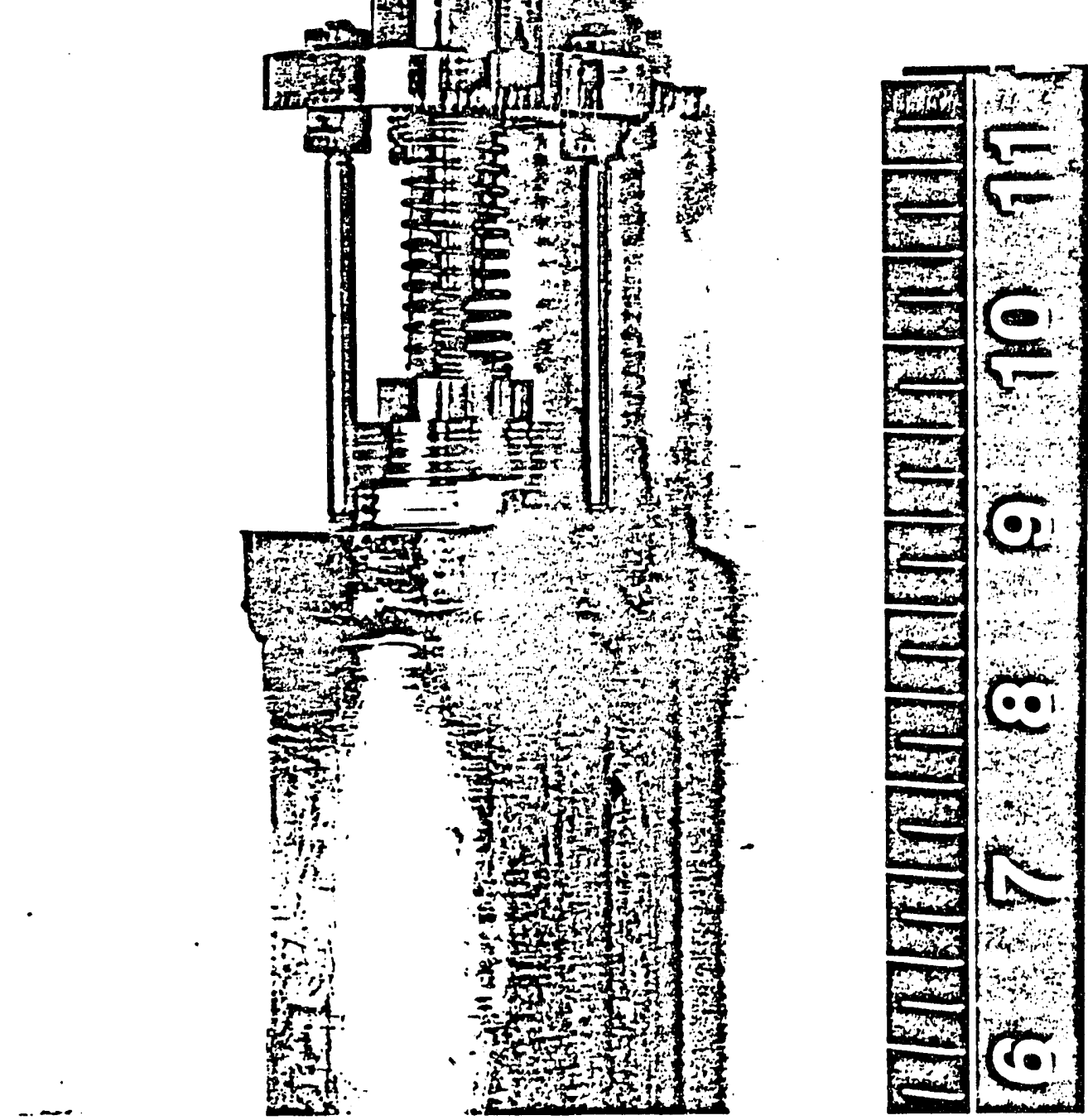

Figure 5 - Top of prototype mechanical sampler with top valve in closed position (Teflon fill plug in foreground). 


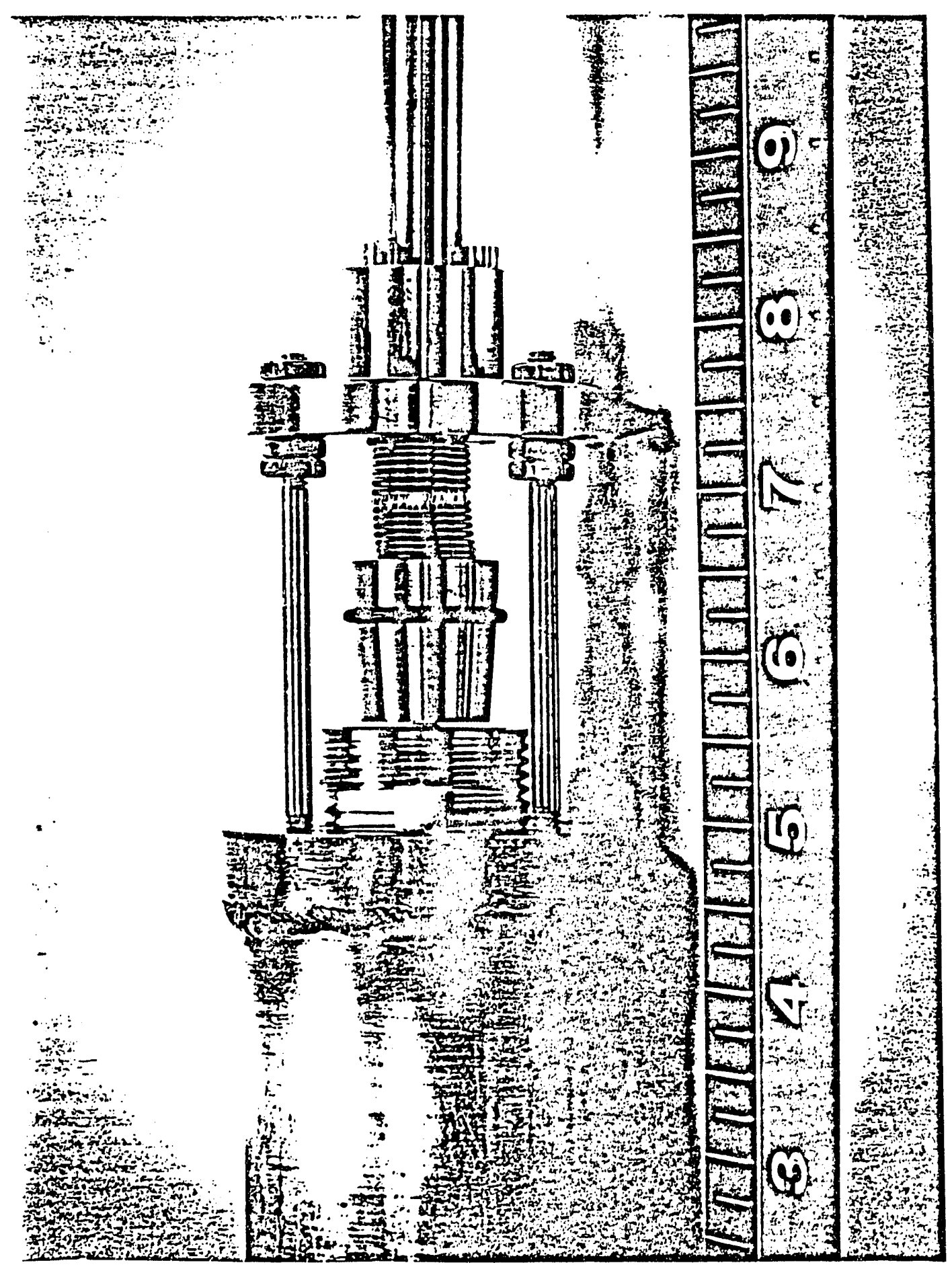

Figure 6 - Top of prototype mechanical sampler with top valve in opened position. 


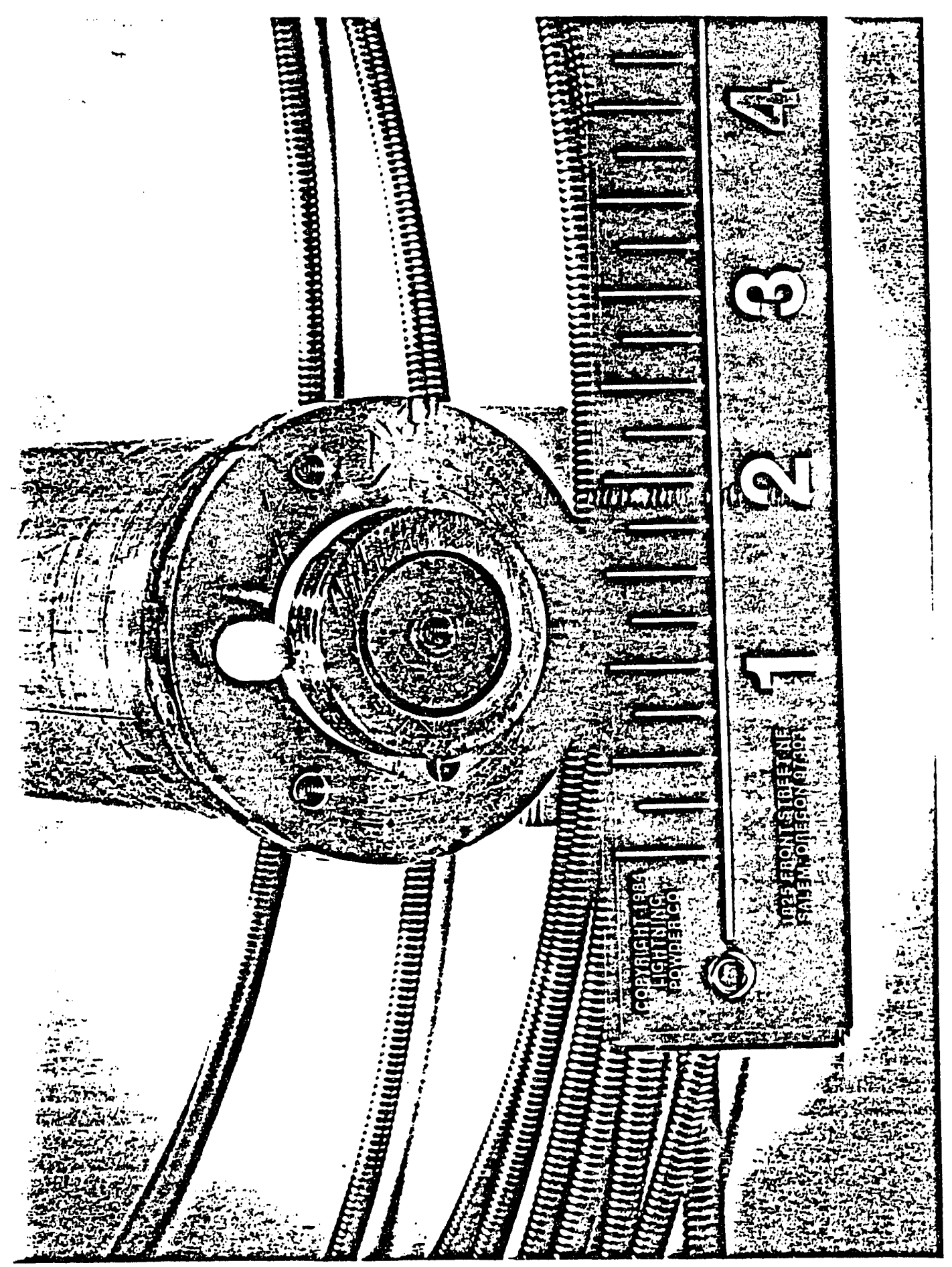

Figure 7 - Bottom of prototype mechanical sampler showing bottom valve in closed position, and Teflon plug for emptying sampler. 


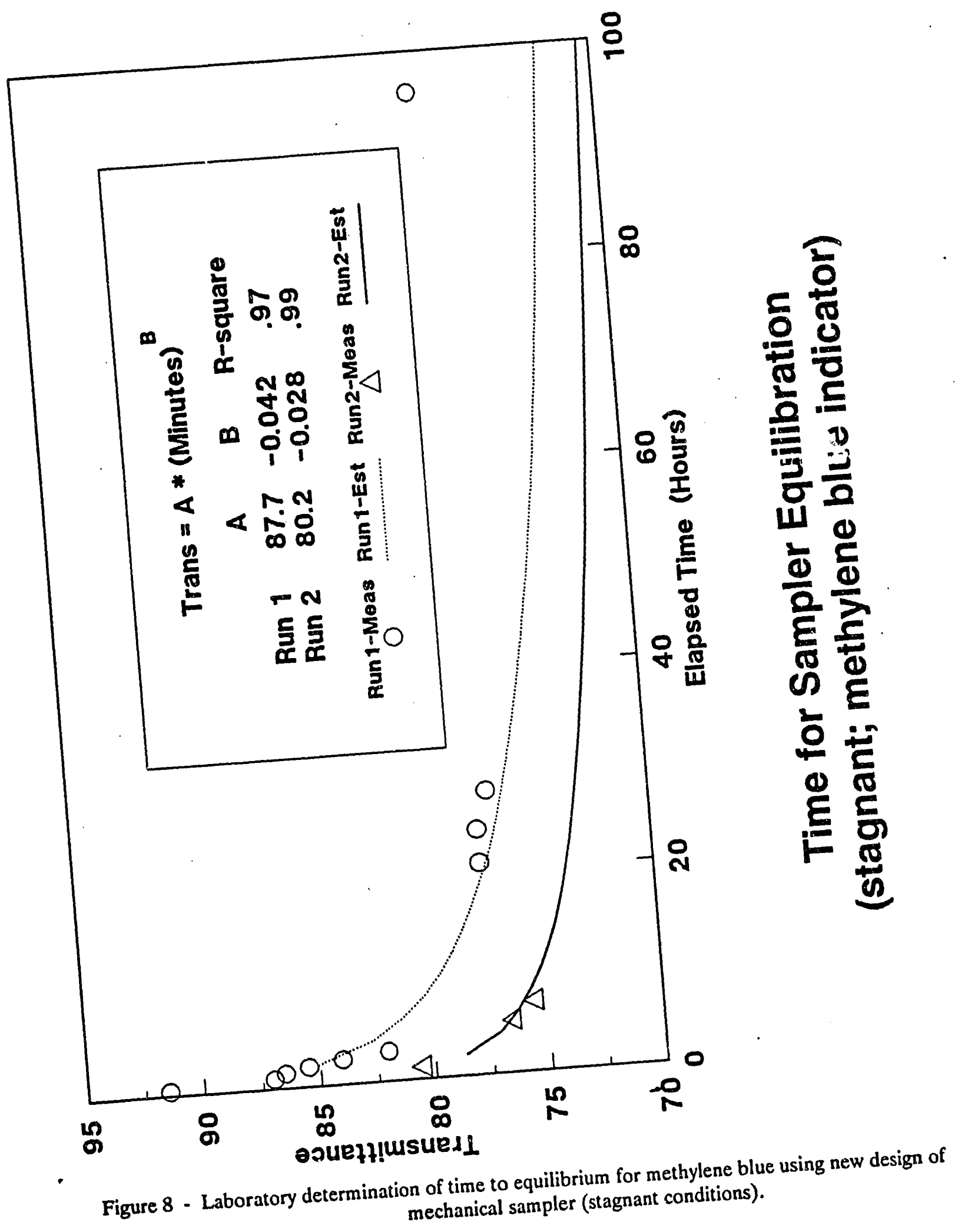




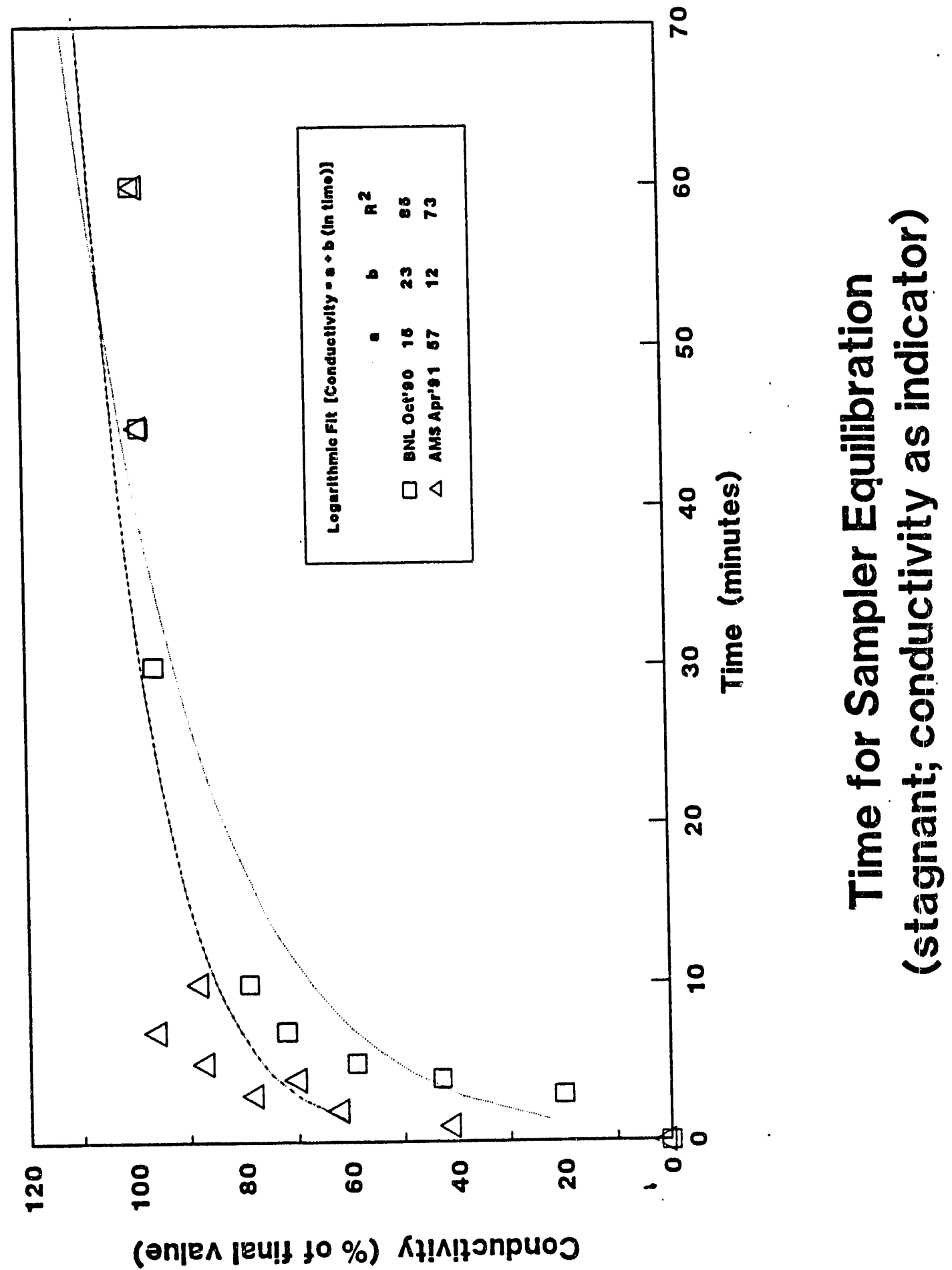

Figure 9 - Laboratory determination of time to equilibrium for the new design of mechanical sampler using conductance as tracer. 


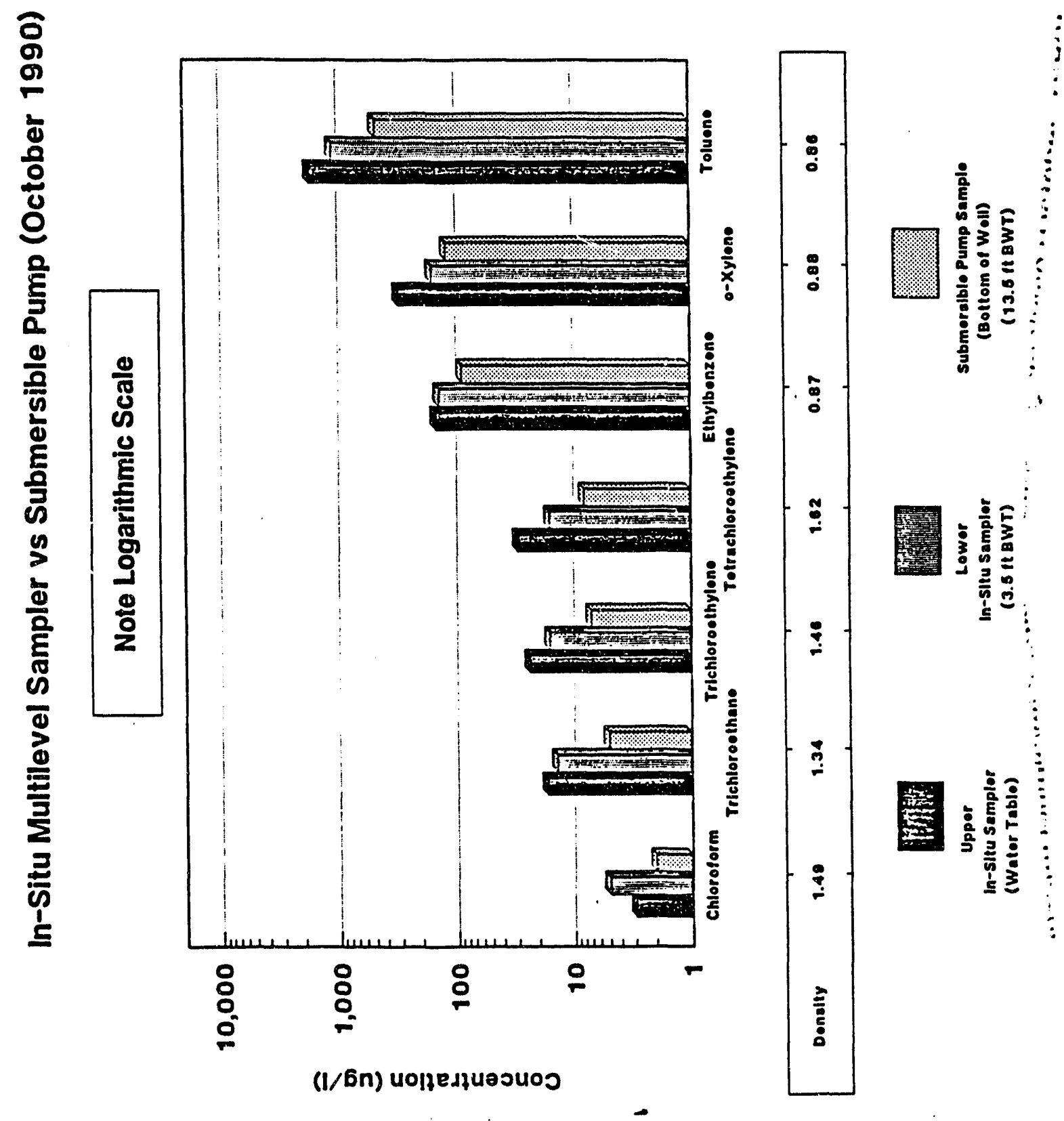

Figure 10 - Results of first field sampling event in comparison to measurements taken with standard BNL sampling protocol. 


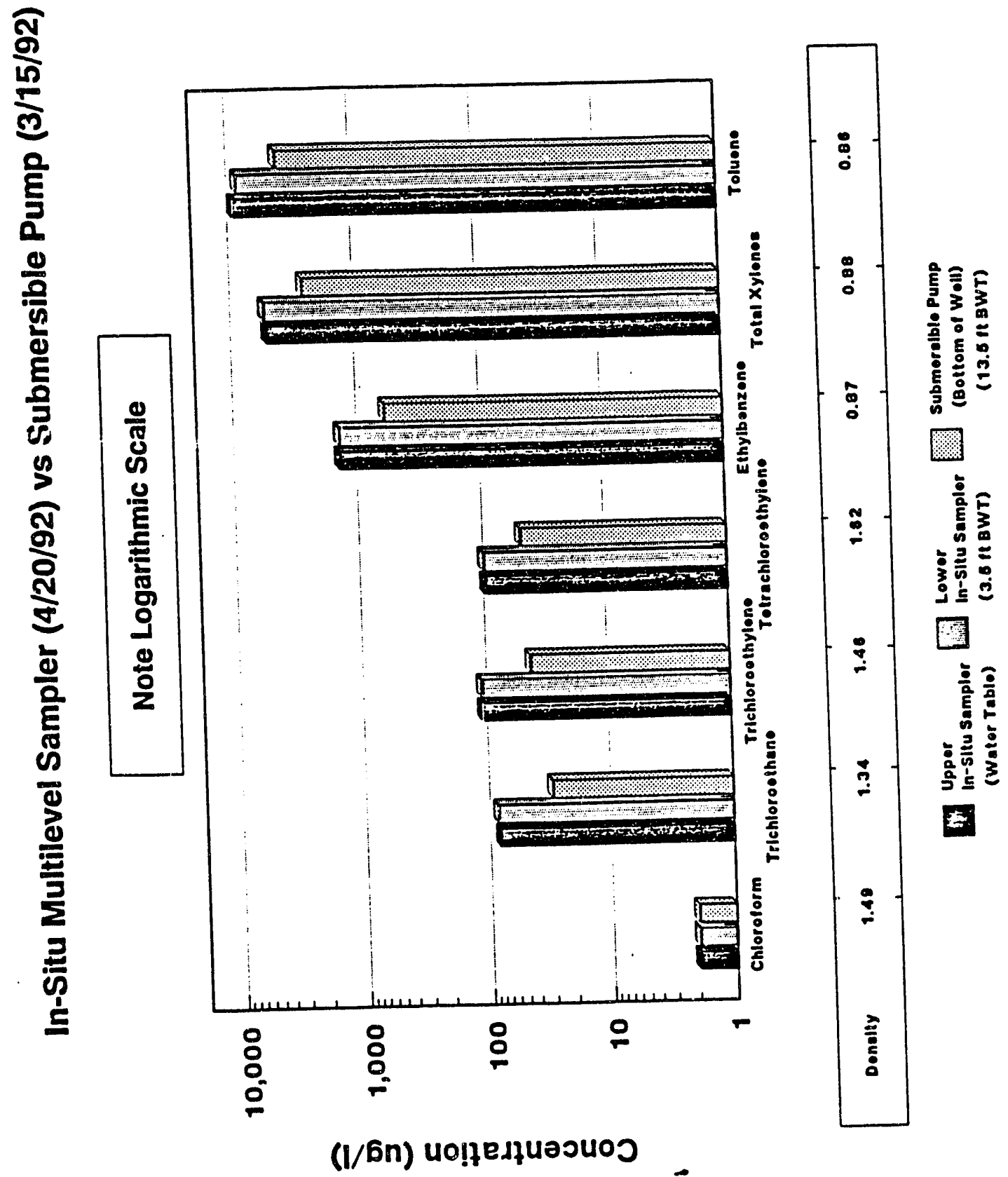

Figure 11 - Results of second field sampling event in comparison to measurements taken with standard BNL sampling protocol. 

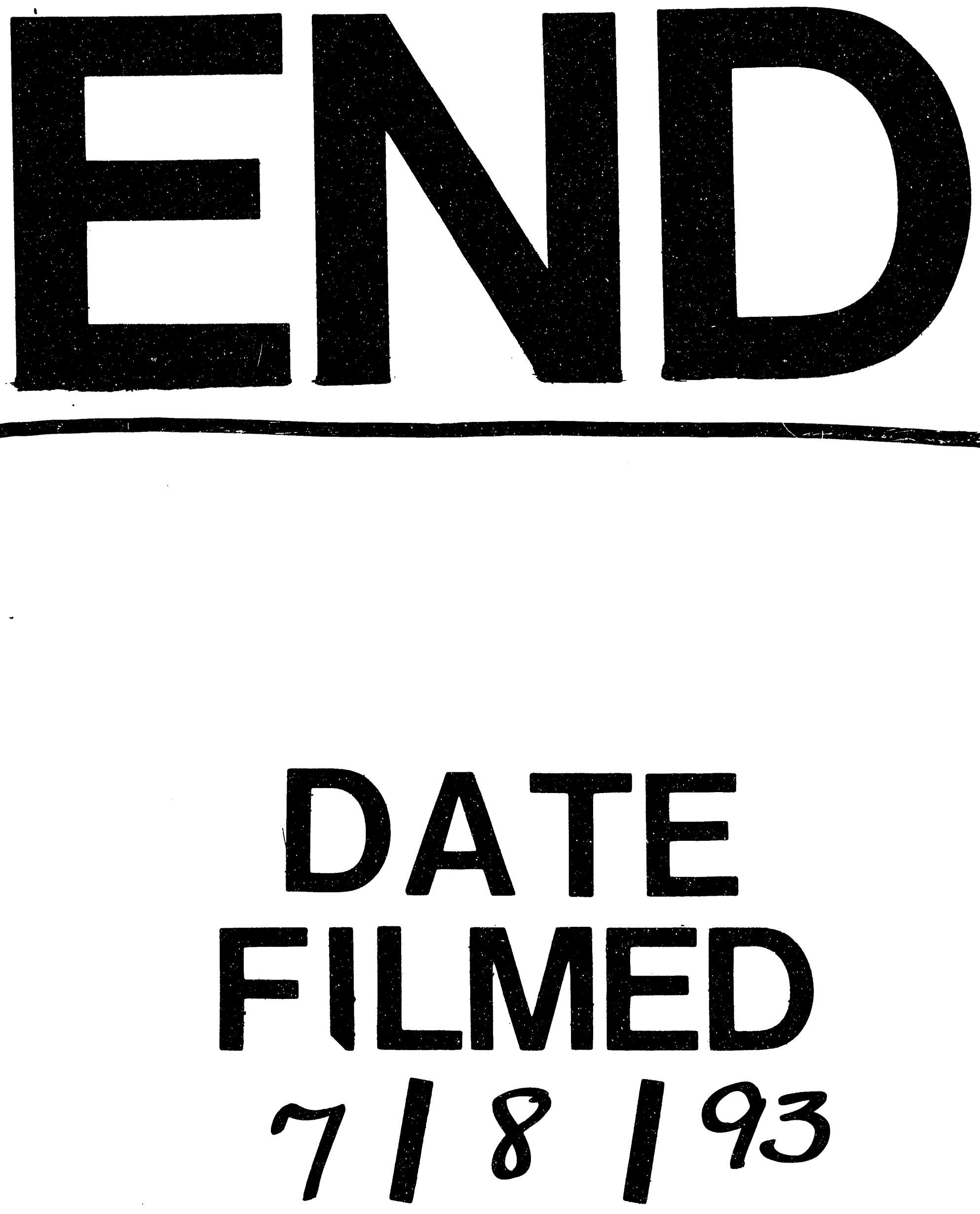
